\title{
Der Übergang in eine nacheheliche Partnerschaft: Eine vergleichende Analyse zwischen Männern und Frauen auf Basis des deutschen Generations and Gender Survey
}

\section{Re-partnering after divorce in Germany - A comparison between men and women based on an analysis with the Generations and Gender Survey}

\begin{abstract}
Zusammenfassung
Angesichts kontinuierlich hoher Scheidungszahlen werden nacheheliche Beziehungen zu regelmäßigen Lebensereignissen innerhalb einer Partnerschaftsbiografie. Allerdings gibt es relativ wenige Studien zu den Determinanten der nachehelichen Paarbildung. In diesem Beitrag werden die Daten des Generations und Gender Surveys (GGS) aus dem Jahr 2005 verwendet, um einen Einblick in das nacheheliche Partnerschaftsverhalten in Deutschland $\mathrm{zu}$ gewinnen. Im Fokus der Betrachtung steht dabei, welche wesentlichen Einflussfaktoren diesen Prozess der Paarbildung bestimmen und zu welchen Unterschieden es dabei zwischen Männern und Frauen kommt. Die Ergebnisse zeigen, dass es bei den Übergangsraten in eine nacheheliche Partnerschaft kaum Geschlechterunterschiede gibt. Sowohl für Männer als auch für Frauen geht vom Bildungsniveau ein positiver Effekt auf die Übergangsrate in eine nacheheliche Partnerschaft aus. Nur in Bezug auf das Alter bei Scheidung gibt es geschlechtsspezifische Unterschiede. Insbesondere bei Frauen zeigt sich der negative Einfluss des Alters deutlicher.
\end{abstract}

Schlagwörter: Nacheheliche Paarbildung, Trennung, Scheidung, Ereignisanalyse

\begin{abstract}
Due to continuously high marital dissolution rates, re-partnering becomes an increasingly regular life course experience. However, only few empirical studies have addressed the topic of repartnering after divorce. This analysis uses data from the first wave of the German Generations and Gender Survey (GGS), which was conducted in 2005, to study patterns of post-divorce union formation in Germany. Particular attention is given to potential gender differences. Surprisingly, the empirical investigation shows no major gender differences in re-partnering rates. High education increases re-partnering rates for both sexes. There is, however, a gender difference for the age at divorce determining re-partnering behaviour: while women who are older at divorce experience a rather low rate of re-partnering, we do not find such a clear pattern for their male counterparts.
\end{abstract}

Key words: post-divorce re-partnering, separation, divorce, event history 


\section{Einleitung}

Im Zuge des familialen Wandels hat sich die Partnerschaftsqualität als das entscheidende Kriterium für die Stabilität von modernen Beziehungen manifestiert (Beck-Gernsheim 1994; Nave-Herz 2004). Die Schwelle dafür, eine Partnerschaft aufzulösen oder gar eine Ehe zu scheiden, ist gesunken, was das Ansteigen der Scheidungsziffern seit den 1970er Jahren zeigt (Lesthaeghe 1992). Trotz abnehmender Partnerschaftsstabilität ist jedoch nicht davon auszugehen, dass ein partnerschaftliches Zusammenleben weniger bevorzugt wird. Da jedoch ein abnehmender Trend in Bezug auf die Wiederheiratshäufigkeit von Geschiedenen zu beobachten ist, wird die nacheheliche Paarbildung scheinbar durch die Zunahme nichtehelicher Lebensgemeinschaften kompensiert (Heekerens 1988: 42). Dieser Zusammenhang lässt sich mit dem analytischen Potenzial der amtlichen Statistik nur sehr schwer beantworten. Anhand der Haushaltsstatistik im Rahmen des Mikrozensus ist allerdings belegbar, dass nichteheliche Partnerschaften neben dem Zusammenleben im früheren Alter auch für nacheheliche Partnerschaften an Bedeutung gewinnen. Ergebnisse zur Schätzung der Paarkonstellationen belegen, dass nichteheliche Partnerschaften mit zwei geschiedenen Partnern einen Anteil von 11 Prozent ausmachen und damit an zweiter Stelle hinter den Partnerschaften mit zwei Ledigen (60 Prozent) liegen (Bundeszentrale für politische Bildung 2008: 29).

Innerhalb der Familienforschung spielt die Analyse des Scheidungsverhaltens schon seit mehreren Jahrzehnten eine zentrale Rolle, jedoch ist das Forschungspotenzial zum nachehelichen Paarbildungsverhalten noch längst nicht erschöpft. Ziel dieses Beitrages ist es, den Einfluss verschiedener Determinanten auf den nachehelichen Paarbildungsprozess zu untersuchen, wobei der Schwerpunkt auf einem Vergleich zwischen Männern und Frauen liegt. Gefragt wird unter anderem, wie lange es nach einer Scheidung dauert, bis jemand mit einem neuen Partner einen gemeinsamen Haushalt teilt. Wie beeinflussen soziodemografische Faktoren wie das Alter oder das Bildungsniveau einer Person diesen Übergangsprozess? Welche Einflüsse sind im Hinblick auf bereits vorhandene Kinder bedeutsam?

Um diese Fragen umfassend zu behandeln, wird zum theoretischen Einstieg in das Thema die Partnerwahl vor dem Hintergrund individueller Entscheidungsmechanismen und struktureller Bedingungen diskutiert. Der Erklärungsgehalt dieser theoretischen Perspektiven wird anschließend auf die Bildung von nachehelichen Partnerschaften projiziert, womit die Ableitung der zentralen Forschungshypothesen im zweiten Abschnitt verbunden ist. Der dritte Abschnitt enthält die methodischen Vorüberlegungen zur empirischen Analyse. Darauf folgt im vierten Abschnitt die Darstellung und Interpretation der Ergebnisse zum Übergang in eine nacheheliche Partnerschaft sowie eine abschließende Reflexion der Studie. 


\section{Theoretische Perspektiven des Partnerwahlverhaltens}

\subsection{Die Partnerwahl mit dem Bezug zur individuellen Handlungsebene}

Für die Erklärung von Partnerwahlprozessen wird immer wieder der klassische Ansatz der ökonomischen Theorie der Familie (Becker 1973, 1993) reflektiert. Aus ökonomischer Perspektive besteht der Vorteil des Zusammenlebens in einer Partnerschaft darin, dass bestimmte Güter kostengünstiger als in einem Einpersonenhaushalt produziert werden können (Becker 1973: 814). Da im Ansatz der „New Home Economics“" die traditionelle Form der Arbeitsteilung zwischen Mann und Frau besonders herausgearbeitet wird, ist die Anwendung der Familienökonomie auf traditionelle Arbeitsmarktstrukturen relativ unproblematisch. Umso mehr stellt sich die Frage, ob die Logik dieser Theorie dem veränderten Geschlechter- und Rollenverhältnis in unseren modernen Gesellschaften noch gerecht wird. Seit dem Beginn des 20. Jahrhunderts haben technologische Entwicklungen, der Ausbau wohlfahrtsstaatlicher Dienstleistungen sowie jüngste Veränderungen der Erwerbsmuster diverse gesellschaftliche Neuerungen ausgelöst, durch die sich das Rollenverständnis der traditionellen Arbeitsteilung grundlegend verändert hat. Geschlechterarrangements, bei denen beide Partner durch Erwerbsarbeit einen Beitrag zum Haushaltseinkommen leisten, sind in den meisten europäischen Ländern längst dominant (Daly 2005: 383). Dieser Wandel lässt sich jedoch in das Grundmodell von Becker integrieren, da die Situation der Arbeitsteilung zur Steigerung der Effizienz des Haushaltes auch dann gegeben ist, wenn beide Partner erwerbstätig sind. Trotz des zunehmenden Anteils der Frauenerwerbstätigkeit hat das ökonomische Modell weiterhin Geltung, da Frauen infolge von Diskriminierungsprozessen auf dem Arbeitsmarkt ein bedeutend geringeres Erwerbseinkommen erzielen als Männer. Zudem unterbrechen sie ihre Erwerbstätigkeit nach der Geburt eines Kindes und übernehmen weitaus mehr Tätigkeiten im Haushalt (Becker 1993: 77).

Ein weiterer mikroanalytischer Ansatz ist die Austauschtheorie, die insbesondere auf familienzyklische Ereignisse anwendbar ist. Der Kerngedanke des austauschtheoretischen Modells hinsichtlich der Partnerwahl erklärt, welche Interaktionen eine Beziehung intensivieren, sodass sich Partner dauerhaft aneinander binden. Die Individuen ziehen anhand der Kosten- und Nutzeneinschätzung ihrer wechselseitigen Tauschbeziehung eine Bilanz und entscheiden sich für eine Fortführung oder einen Abbruch der Beziehung (Blau 1967: 92f.), wobei in einer familiären Austauschbeziehung der Wert einer erhaltenen Leistung nicht in gleichem Maße zurückgefordert wird (Nye 1979: 10). Ein wesentlicher Vorzug der austauschtheoretisch orientierten Erklärungsversuche ist die dynamische Betrachtungsweise. Die Paarbildung verläuft als kontinuierlicher Interaktionsprozess, indem zwischen den Akteuren ein Austausch von Ressourcen stattfindet, der mit der Situation und über die Zeit variiert. Ein weiterer positiver Aspekt des austauschtheoretischen Ansatzes ist die Erklärung, warum andere Handlungsoptionen trotz scheinbarer Vorteile nicht gewählt werden (Thibaut/Kelley 1959: 24). Im Gegensatz zum familienökonomischen ist die austauschtheoretische Perspektive nicht auf einen gemeinsamen Haushalt des Paares festgelegt. Dieser Zusammenhang könnte für nacheheliche Partnerschaften von besonderer Relevanz sein, da Partnerschaften ohne gemeinsames Zusammenleben eventuell bevorzugt werden - auch aufgrund der negativen Erfahrung, die mit der Auflösung des ehemaligen ehelichen Haushaltes verbunden ist. 
Obwohl die Austauschtheorie viel breiter angelegt ist, basieren die folgenden Herleitungen auf familienökonomischen Überlegungen. Vor dem Hintergrund, dass in dieser Untersuchung der Zusammenzug beider Partner das zentrale Ereignis darstellt, erscheint die Fokussierung auf den Haushaltskontext als eine angemessene Vorgehensweise zur theoretischen Modellierung. Becker projiziert seine ökonomischen Überlegungen auf den Haushaltskontext, in dem zwei Partner miteinander in Kooperation stehen - „[...] persons in love can reduce their costs of frequent contact and of resource transfers by sharing the same household“ (Becker 1973: 819). Damit ist eine Generalisierbarkeit des partnerschaftlichen Zusammenlebens möglich, und die Partnerwahl kann somit auch für geschiedene Personen vor dem Hintergrund der Familienökonomie betrachtet werden, solange mit der nachehelichen Beziehung eine gemeinsame Haushaltsführung verbunden ist. Die Theorie postuliert dann einen maximalen Gewinn aus der gemeinsamen Verbindung, wenn die Frau in haushaltsspezifisches und der Mann in marktspezifisches Humankapital investiert. Nach diesem traditionellen Konzept haben geschiedene Personen über einen mehr oder weniger langen Zeitraum ehedauerspezifisches Kapital angesammelt. Frauen, die in der Ehe ihre arbeitsmarktspezifischen Qualifikationen zugunsten der traditionellen Arbeitsteilung vernachlässigt und hauptsächlich in haushaltsbezogenes Kapital investiert haben, werden nach der Scheidung schlechtere Chancen auf dem Arbeitsmarkt haben. Diese unsichere ökonomische Situation erschwert es ihnen, die negativen Konsequenzen einer Scheidung zu überwinden, was in den meisten Fällen nur durch eine Folgepartnerschaft gelingt (De Graaf/Kalmijn 2003: 1460). Dementsprechend liegt die Motivation, eine neue Partnerschaft zu gründen, in erster Linie darin, die eigene finanzielle Absicherung zu gewährleisten. Analog kann diese Überlegung auf Männer übertragen werden, wenn diese eine einseitige Investition in erwerbsorientiertes Humankapital vorgenommen und weniger ihre Fähigkeiten bezüglich einer effektiven Haushalts- und Erziehungsarbeit ausgebaut haben. In diesem Fall besteht auch für geschiedene Männer eine starke Orientierung auf ein partnerschaftliches Zusammenleben. Eine andere Situation ergibt sich für geschiedene Männer, die nach der Scheidung finanzielle Belastungen für die ehemalige Familie tragen. Hier stellt sich die Frage, wie attraktiv eine neue Partnerin ist, die kein monetäres Einkommen erzielen kann. Will und kann ein geschiedener Mann dann noch die alleinige Rolle des Familienversorgers übernehmen? Zu diesem Aspekt sind Oppenheimers Überlegungen (Oppenheimer 1988, 1994) relevant. Neben der Auflösung traditioneller Geschlechterrollen müssen auch die veränderten Arbeitsmarktstrukturen seit den 1980er Jahren berücksichtigt werden. In Zeiten diskontinuierlicher Erwerbsverläufe aufgrund von schlechteren Arbeitsmarktbedingungen wird eine Investition beider Partner in marktspezifisches Kapital notwendig, um die Phase einer eventuellen Arbeitslosigkeit des einen Partners zu überbrücken und den Einkommensausfall abzufedern (Oppenheimer 1994: 318). In Bezug auf die Gründung von Folgepartnerschaften kann daraus abgeleitet werden, dass für besser ausgebildete Frauen zwar kein ökonomischer Zwang zur Verpartnerung besteht, was die Notwendigkeit einer erneuten Paarbildung einerseits verringert, andererseits jedoch mit einer Erhöhung ihrer Attraktivität einhergeht (Oppenheimer 1988). 


\subsection{Strukturbedingte Mechanismen des Partnerwahlprozesses}

Betrachtet man die Aspekte der Partnerwahl in Abhängigkeit von den Mechanismen des Partnermarkts, schränkt dieser als Ort der Begegnung die Partnerwahl sowohl räumlich als auch sozial ein. Nach dem strukturtheoretischen Ansatz wird anhand der sozialen Positionen, die von Personen eingenommen werden, eine gesellschaftliche Schichtung erzeugt (Blau 1977: 28). Je größer die Statusdistanz zwischen zwei Personen, desto geringer ist die Wahrscheinlichkeit ihres Zusammentreffens (Blau 1994: 30ff.). Nach dem Prinzip "Who does not meet does not mate" erfolgt die Herstellung von Interaktionsgelegenheiten innerhalb bestimmter „Verkehrskreise“ (Lengerer 2001: 151). Eingegrenzt werden diese durch die soziale Umgebung sowie durch regionale und kulturelle Aspekte, die einen bedeutsamen Platz im Leben einer Person einnehmen (Nave-Herz 2004: 133). Für die ausgesuchte Untersuchungspopulation der Geschiedenen scheint das Arbeits- und Berufsumfeld besonders relevant. Aufgrund der lebenszeitlichen Verankerung des Scheidungsereignisses haben diese Personen in den meisten Fällen ihre Ausbildungsphase längst abgeschlossen. Neben den Entwicklungen wie dem Anstieg des Alters bei Erstheirat führt außerdem die kontinuierliche Zunahme der Frauenerwerbsbeteiligung dazu, dass die Bedeutung des Arbeitsplatzes als lokaler Partnermarkt zugenommen hat und sich viele Partner am Arbeitsplatz kennen lernen (Hochschild 1997: 201).

Im Zusammenhang mit den Einflussfaktoren des Partnermarkts ist die bevölkerungsstrukturelle Komponente ein weiterer nicht zu vernachlässigender Faktor. Demografische Strukturen, die mit einem numerischen Ungleichgewicht zwischen Männern und Frauen einhergehen, können erhebliche Konsequenzen für die Partnerwahl haben. In der sozialwissenschaftlichen Literatur wird dieser Effekt hinsichtlich der rein quantitativen Verteilung zwischen den potenziellen Partnern mit dem Begriff „marriage squeeze“ bezeichnet (Akers 1967; Schoen 1983). Auch für das Scheidungs- und Wiederverheiratungsverhalten sind die Bedingungen des Partnermarkts von zentraler Bedeutung. Wenn nicht genügend Personen des anderen Geschlechts zur Verfügung stehen, werden die strukturbedingten Zwänge eine bestimmte Partnerkombination oder gar Partnerlosigkeit für gewisse Gruppen zur Folge haben (Klein 2000). Es ist jedoch schwierig zu beurteilen, ob ein günstiger Erst-Heiratsmarkt gleichzeitig auch günstige Bedingungen für die Partnerwahl der selektiven Gruppe der Geschiedenen bereitstellt. Muster der Partnerwahl können mitunter von lokalen Bedingungen modifiziert werden. Auch wenn geschiedene Personen aufgrund ihres fortgeschrittenen Alters bildungs- und altershomogene Teilpartnermärkte verlassen haben, können diese Mechanismen durch berufliche oder freundschaftliche Netzwerke kompensiert werden, indem diese Institutionen positive Bedingungen für das Zusammentreffen von potenziellen Partnern schaffen.

Insgesamt betrachtet ist die Partnerwahl für geschiedene Personen mit einer zunehmenden Kompromissbereitschaft verbunden, wodurch nacheheliche Partnerschaften in einigen Merkmalen eher von Heterogamie geprägt sein können. So verringert sich die Altershomogamie zwischen Personen, die schon einmal verheiratet waren (South 1991: 929; Booth-Edwards 1992: 192). Da sich Partnermarktstrukturen altersspezifisch verändern, sind diese für das Paarbildungsverhalten von Geschiedenen zu berücksichtigen. Mit steigendem Alter wird der Partnermarkt kleiner und ineffizienter, weil ein großer Anteil von potenziellen Partnern im altersnahen Bereich partnerschaftlich fest gebunden 
ist. ${ }^{1}$ Aus Sicht der Lebenslaufperspektive verschlechtern sich die Aussichten auf dem Partnermarkt zunehmend. Numerische Ungleichgewichte sind unter potenziellen Partnern mit gleichen Eigenschaften oft umso ausgeprägter, je älter die Personen sind, womit sich außerdem die abnehmenden Homogamiequoten erklären lassen (Klein 1999: 231f.).

\subsection{Ableitung der zentralen Forschungshypothesen}

Der vorliegende Beitrag fokussiert auf Geschlechterunterschiede im nachehelichen Partnerbildungsverhalten. Auf Basis der Vorüberlegungen liegt es nahe zu vermuten, dass diese Geschlechterunterschiede mit dem Alter und der Bildung der Befragten in Verbindung stehen. Aufgrund der an das Lebensalter geknüpften Mechanismen bietet das Alter zum Auflösungszeitpunkt der Ehe eine sehr wichtige Orientierung für die Erklärung des nachehelichen Paarbildungsverhaltens, nämlich mit Bezug auf die Verfügbarkeit potenzieller Partner. Mit steigendem Alter schmilzt nicht nur der „Partnerpool“, auch die physische Attraktivität einer Person nimmt ab und die gesundheitlichen Beeinträchtigungen steigen, was die eigene Position auf dem Partnermarkt verschlechtert. Somit ist von einem negativen Alterseffekt auszugehen, das heißt: Mit steigendem Alter bei Auflösung der Ehe sinken die Chancen für die Gründung einer nachehelichen Partnerschaft (Hypothese 1). Dieser Effekt auf das nacheheliche Paarbildungsverhalten wird für Frauen stärker sein als für Männer (Bumpass/Sweet/Martin 1990). Mit zunehmendem Alter verschlechtert sich die Position auf dem Partnermarkt für geschiedene Frauen drastischer, zum einen weil geschiedene Männer im „Wiederverpartnerungsprozess“ auf ein viel weiteres Altersspektrum zurückgreifen und mitunter jüngere Partnerinnen bevorzugen (Hughes 2000). Andererseits verschiebt sich mit zunehmendem Alter das Geschlechterverhältnis, was mitunter für Frauen verminderte Chancen zur Paarbildung zur Folge hat (Engstler 2003: 206).

Beim Paarbildungsverhalten von geschiedenen Personen muss vor allem die ökonomische Situation vielschichtig beleuchtet werden, da sich diese aufgrund der getätigten Investitionen in der vorherigen Ehe, beispielsweise gemeinsame Kinder oder Wohneigentum, oft kompliziert gestaltet. Als empirisch messbarer Faktor für den sozioökonomischen Status ist das Bildungsniveau einer Person von zentraler Bedeutung. Dahingehend lässt sich folgender Zusammenhang vermuten: Je größer das Ausmaß bildungsspezifischer Ressourcen, desto höher die Chancen für das Eingehen einer nachehelichen Partnerschaft (Hypothese 2). Es ist davon auszugehen, dass ein hohes Ausbildungsniveau außer mit steigenden Einkommenschancen auch mit einer höheren Wahrscheinlichkeit für eine Erwerbsbeteiligung einhergeht. Diese Faktoren tragen entscheidend zur individuellen Attraktivität einer Person auf dem Partnermarkt bei. In Bezug auf die strukturellen Mechanismen der Partnerwahl erzeugt die Partizipation am Arbeitsmarkt zudem günstige Rahmenbedingungen für das Zusammentreffen mit potenziellen Partnern (Hughes 2000). Hinsichtlich der Geschlechterabhängigkeit des Bildungseffektes ist ein positiver Einfluss sowohl bei Männern als auch bei Frauen zu erwarten. Frauen, die ein hohes Bildungsni-

1 Zwar stehen Geschiedene als selektive Gruppe dem Partnermarkt wieder uneingeschränkt zur Verfügung, jedoch kommen auch ,partnerschaftlich gebundene Personen“ mit einer bestimmten, wenn auch geringen Wahrscheinlichkeit für eine Folgebeziehung in Frage (Stauder 2006). 
veau erreicht haben, also besser mit Humankapital ausgestattet sind, haben größere Chancen auf dem Partnermarkt (Oppenheimer 1988). Zwar postuliert das familienökonomische Prinzip für niedrig gebildete Frauen eine höhere Neigung zur nachehelichen Paarbildung, jedoch ist davon auszugehen, dass die schwindende Attraktivität aufgrund der ökonomisch schlechter gestellten Situation die Wirkung individueller Präferenzen überlagert. Bedenkt man außerdem, dass die negativen wirtschaftlichen Folgen einer Scheidung häufig durch staatliche Transferleistungen kompensiert werden, lässt sich auch aus diesem Grund für niedrig gebildete Frauen eine geringere Neigung zum nachehelichen Paarbildungsverhalten herleiten - um nämlich den Anspruch auf Sozialtransfers nicht durch ein gemeinsames Haushaltseinkommen zu verwirken (De Graaf/Kalmijn 2003). Männer, die weniger ökonomische Ressourcen zur Verfügung haben, können nach der Scheidung in eine prekäre finanzielle Situation geraten, wodurch eine nacheheliche Haushaltsgründung zunehmend erschwert werden könnte. Jedoch sind Männer zu einem höheren Anteil erwerbstätig und seltener von Sozialtransfers oder Unterhaltszahlungen abhängig.

Inwieweit die Kinder aus der geschiedenen Ehe den Übergang in eine neue Partnerschaft verhindern oder begünstigen ist ein vielfach diskutierter Zusammenhang. Wenn Kinder im Haushalt leben ist die Gründung einer nachehelichen Partnerschaft auch immer mit der Gründung einer Stieffamilie verbunden, die alle Beteiligten vor eine Herausforderung stellt (Textor 2010). Es lässt sich annehmen, dass für das Zusammenziehen mit einem neuen Partner bereits im Haushalt lebende Kinder eine größere Barriere für den betreuenden Elternteil darstellen (Bernhardt 2000): Je mehr Kinder zum Zeitpunkt der Scheidung im Haushalt sind, desto mehr verstärkt sich der hemmende Effekt auf das nacheheliche Paarbildungsverhalten (Hypothese 3a). Da die Betreuungsperson der gemeinsamen Kinder stärker an den Haushalt gebunden ist, werden zudem die Kontaktmöglichkeiten zu potenziellen Partnern aufgrund einer gewissen sozialen Isolation eingeschränkt (Klein 1990). Neben der Anzahl der Kinder im Haushalt sollte man auch das Alter der Kinder betrachten. Die Begründung liegt darin, dass jüngere Kinder einen höheren Betreuungsbedarf fordern, der beide Elternteile involviert. Zwar hat der Elternteil, bei dem die Kinder nicht leben, ein völlig unterschiedliches Ausmaß an Betreuungszeit, jedoch kann bei einer Aufrechterhaltung der Bindung durch regelmäßige, aber dafür sehr intensive Besuche der Kinder eine neue Partnerschaft trotzdem erschwert werden (Textor 2010): Dementsprechend ist ein negativer Effekt bezüglich des Alters des jüngsten Kindes zu erwarten, da im jüngeren Kindesalter mehr Betreuungsaufwand durch die Elternteile erforderlich ist (Hypothese 3b).

Zusammenfassend lässt sich aus den dargestellten Überlegungen als allgemeine Hypothese zum Einfluss des Geschlechts formulieren, dass Männer häufiger eine nacheheliche Partnerschaft gründen werden als Frauen (Hypothese 4). Diese Vermutung begründet sich einerseits aus der angeführten Geschlechterabhängigkeit bezüglich des Alterseffektes, anderseits ist dem Einfluss von Kindern eine entscheidende Bedeutung in Bezug auf die Erklärung von Geschlechterunterschieden beizumessen - dies scheint für Frauen stärker ins Gewicht zu fallen, da nach einer Scheidung die Kinder überwiegend im mütterlichen Haushalt leben (Tammen 2007: 529ff.). Vergleichbare Studien zeigen, dass unter Geschiedenen letztendlich mehr Frauen alleine leben als Männer (Wu/Balakrishan 1994; Hughes 2000; Wu/Schimmele 2005). 


\section{Vorüberlegungen zur empirischen Analyse}

\subsection{Erklärungen zur Methode}

Die empirische Untersuchung wird mit Hilfe von ereignisanalytischen Verfahren vorgenommen. Dazu müssen Längsschnittdaten vorliegen, die sich auf bestimmbare Individuen beziehen und über die Zeit variieren (Blossfeld et al. 2007). In dieser Analyse wird auf einen ganz bestimmten Zeitraum einer Partnerschaftsbiografie fokussiert, der mit der Auflösung einer Ehe beginnt und mit der Gründung einer nachehelichen Partnerschaft endet. Damit stellt der Übergang in eine nacheheliche Partnerschaft das zu erklärende Ereignis dar. Um Verweildaueranalysen vornehmen zu können, muss die Struktur eines Ereignisdatensatzes immer eine Zeit- und Zensierungsvariable beinhalten. In diesem Zusammenhang wird die Prozesszeit folgendermaßen definiert: tritt das Ereignis ein, so bezieht sich die gemessene Verweildauer auf die Differenz zwischen dem Zeitpunkt des Zusammenzugs mit dem neuen Partner und dem Auflösungszeitpunkt der vorherigen Ehe; erfährt die befragte Person das Ereignis nicht, so ergibt sich die Prozesszeit aus der Differenz zwischen dem Interview- und dem Scheidungszeitpunkt.

Für die Bestimmung eines multivariaten Modells ist die Verteilung des Ereignisses im Zeitverlauf relevant. Ein geeignetes Modell für die Möglichkeit der Schätzung ist das Piecewise Exponential Model, mit dem sich die Basisprozesszeit näher spezifizieren lässt. Entsprechend der Modellannahme erfolgt eine Unterteilung der Risikozeit in festgelegte Segmente. Das Risiko kann also nur zwischen den einzelnen Intervallen variieren und ist innerhalb eines Intervalls immer konstant (Blossfeld/Rohwer 2002: 120-130). Das Modell lässt sich mathematisch folgendermaßen beschreiben:

$$
h(t \mid Z)=h_{0}(t) \exp \left(\sum_{k=1}^{p} \beta_{k} Z_{k}\right)
$$

Dabei beschreibt $h_{0}(t)$ die Baseline-Hazardfunktion für das Eintreten des zu untersuchenden Ereignisses zum Zeitpunkt $(t)$. Die Übergangsrate wird durch die Basisübergangsrate $h_{0}(t)$ sowie durch die unbekannten, zu schätzenden Parameter $\left(\beta_{k}\right)$ und die bekannten Kovariaten $\left(Z_{k}\right)$ beeinflusst. Die Parameter werden anhand der Maximum-Likelihood-Methode geschätzt.

\subsection{Beschreibung des Datensatzes und der Datenselektion}

Für die empirischen Analysen werden die Daten des deutschen Generations and Gender Surveys (GGS) aus dem Jahr 2005 herangezogen. Diese Umfrage ist eine international durchgeführte Erhebung mit dem Ziel, vergleichbare Analysen und Ergebnisse zum Thema Familiengründungs- und Geburtenverhalten bereitzustellen. Neben dem generativen Verhalten und verschiedenen familienbezogenen Ereignissen sind Geschlechter- und Generationenbeziehungen ein weiterer Schwerpunkt im GGS, wodurch vielschichtige Untersuchungen zu Paarbildungs- bzw. Paarauflösungsprozessen angestellt werden können (Ruckdeschel et al. 2006: 7f.). Die Datenstruktur des GGS ermöglicht es, eine umfassende 
Partnerschaftsbiografie der Befragten zu konstruieren. Die aktuelle Partnerschaft wird über das so genannte Haushaltsraster erfasst. Daraus wird ersichtlich, ob die Person mit ihrem Partner einen gemeinsamen Haushalt teilt oder ob es sich um eine intime Beziehung handelt, bei der die Partner aber nicht zusammenwohnen. Vorherige Partnerschaften werden ebenfalls detailliert registriert. Damit werden zu allen Partnerschaften einer Person chronologisch und monatsgenau die verschiedenen Ereignisse wie Zusammenzug mit dem Partner, Heirat und Auflösung der Partnerschaft durch Trennung, Scheidung oder Verwitwung erfasst. ${ }^{2}$

Insgesamt wurden 10.017 in Privathaushalten lebende deutschsprachige Personen befragt, die zum Zeitpunkt des Interviews zwischen 18 und 79 Jahre alt waren. Für die Auswahl der Stichprobe kommen nur Personen in Frage, die mindestens eine Scheidung erlebt haben. Im Prozess der Datenselektion wurden folgende Fälle aus der Analyse ausgeschlossen: keine Angaben zum Zusammenzug mit einem neuen Partner, keine Informationen weder zum Trennungs- noch zum Scheidungszeitpunkt, bei unlogischer Reihenfolge der Ereignisse, wenn der Partner verstorben ist oder Befragte außerhalb der Altersspanne 18-65 Jahre bei Auflösung der Ehe liegen. Somit verbleiben 338 Männer und 509 Frauen im Auswahlsample. Von den insgesamt 847 Personen sind 485 Befragte eine nacheheliche Beziehung eingegangen, 362 Personen sind zensiert, da sie das Ereignis bis zum Zeitpunkt der Befragung nicht erfahren (siehe Anhang, Tabelle A1).

\subsection{Operationalisierung der Variablen}

\section{Abhängige Variable}

In dieser Untersuchung wird der Beginn einer nachehelichen Partnerschaft durch das $\mathrm{Zu}$ sammenziehen in einen gemeinsamen Haushalts bestimmt. Eine solche Vorgehensweise berücksichtigt nicht jene Personen, die nach ihrer Scheidung eine Folgebeziehung eingehen, aber nicht zusammen leben. Diese Einschränkung ergibt sich aus der Datenstruktur, da im GGS nur Partnerschaften gezählt werden, wenn die Partner in einem Haushalt zusammengelebt haben. Basierend auf der theoretischen Argumentation fokussiert diese Analyse zum Übergang in eine nacheheliche Partnerschaft auf die Gründung eines gemeinsamen Haushaltes. In der Realität lässt sich der Beginn einer Partnerschaft oft schwer definieren, da Paarbildungen immer mit einem individuellen Entwicklungsprozess verknüpft sind, der graduell verläuft. Dagegen stellt das Zusammenziehen mit dem Partner ein quantifizierbares Ereignis da, das auch den Institutionalisierungsgrad einer Partnerschaft enorm erhöht. Zur genauen Bestimmung der Prozesszeit müssen folgende Annahmen getroffen werden: Aufgrund der rechtlichen Bedingungen des deutschen Scheidungsrechts, das die Einhaltung eines Trennungsjahres vorsieht, geht einer Scheidung die Trennung fast immer voraus. Auch wenn erst das Scheidungsdatum die Ehe rechtmäßig auflöst, scheint es für diese Untersuchung angemessen, als Startzeitpunkt der Betrachtung

2 Als Grundlage für die Berechnungen diente eine bereinigte Version des Datensatzes. Im Rahmen des „Nonmarital Childbearing Projects“ am Max-Planck-Institut für demografische Forschung wurden die GGS Daten mehrerer Länder hinsichtlich der Fertilitäts- und Partnerschaftsbiographien einheitlich aufbereitet (vgl. Perelli-Harris et. al. 2009). 
das Trennungsdatum zu wählen. ${ }^{3}$ Nur wenn die Angaben zum Trennungszeitpunkt unvollständig sind, wird auf das Scheidungsdatum zurückgegriffen. ${ }^{4}$ Bei einigen Personen sind die relevanten Ereignisse auf den gleichen Zeitpunkt datiert. Diese Unschärfen sind höchstwahrscheinlich dem retrospektiven Charakter der Befragung geschuldet, da in der Vergangenheit liegende Erfahrungen im Nachhinein nicht immer realitätsgetreu wiedergegeben werden. Außerdem nehmen Partnerschaften im Privatleben der Menschen einen sehr intimen Bereich ein. Allein die Sensibilität des Themas ist ein entscheidender Aspekt der Reliabilitätsproblematik (Diekmann 2004: 383). Um eine Prozesszeit von null zu vermeiden, wurde in solchen Fällen zwischen dem Trennungsdatum und dem Zeitpunkt des Zusammenzugs mit dem Folgepartner ein Zeitraum von einem Monat unterstellt. ${ }^{5}$ Diese Korrektur in den Daten dient dem Zweck, diese problematischen Fälle nicht aus der Untersuchung ausschließen zu müssen. Der Nachteil dieser Handhabung besteht allerdings in einer manuell herbeigeführten Verzerrung der Ergebnisse, die eine Beschleunigung des zu untersuchenden Übergangs am Anfang des Prozesses bewirken könnte.

\section{Unabhängige Variablen}

Die Analyse der Determinanten des Übergangs in eine nacheheliche Partnerschaft schließt neben den Haupteinflussfaktoren wie Trennungsalter, Bildung, Anzahl der Kinder im Haushalt, Alter des jüngsten Kindes sowie Geschlecht und weitere Kovariaten wie Religiosität und Wohnregion mit ein (siehe Anhang, Tabelle A2). Die Generierung der Bildungsvariablen erfolgt anhand des erreichten Bildungsabschlusses. Dabei werden drei Bildungsgrade unterschieden: „niedrig“ (Haupt- bzw. Volksschulabschluss oder polytechnische Oberschule mit Abschluss der 8./9. Klasse), „mittel“ (mittlere Reife, Realschulabschluss oder polytechnische Oberschule mit Abschluss der 10. Klasse) und „hoch“ (Fachhochschulreife und allgemeine oder fachgebundene Hochschulreife). Es wird unterstellt, dass der Bildungsabschluss bereits vor den relevanten Ereignissen wie Scheidung oder neue Partnerschaft erreicht wurde. Um den Einfluss von Kindern zu messen, werden zwei Variablen konstruiert, die als zeitunabhängige Kovariaten die Information zum Zeitpunkt der Trennung wiedergeben. Die Variable Anzahl der Kinder im Haushalt zum Trennungszeitpunkt hat vier Ausprägungen (,gar keine Kinder“, „keine Kinder im Haushalt“, ,ein Kind im Haushalt" und „zwei oder mehr Kinder im Haushalt"). Das Alter des jüngsten Kindes wird über die vier Ausprägungen „kein Kind“, „0-9 Jahre“, „10-17 Jahre“ und „18 und älter" kategorisiert. Im Gegensatz zur Kinderzahl im Haushalt liefert das Alter des letztgeborenen Kindes eine wichtige Information zum Ausmaß der elterlichen Verantwortung. Diese Variable misst somit den Einfluss von Kindern unabhängig davon, ob sie im Haushalt leben oder nicht.

Ein weiterer Bestimmungsfaktor für die Analyse ist der Einfluss der Religion. Der Bezug zur Religiosität einer Person wird über die Häufigkeit der Teilnahme an religiösen Veranstaltungen abgeleitet und bezieht sich nicht auf eine bestimmte Konfessionszuge-

3 Dieses methodische Vorgehen deckt sich mit dem anderer Studien zum Thema „Remarriage“ (Bumpass et al. 1990) oder „Repartnering“ (Wu/Schimmele 2005).

4 Für insgesamt 9 Fälle wird bei der Berechnung der Prozesszeit sowie des Alters bei Auflösung der Ehe vom Zeitpunkt der Scheidung ausgegangen, da es keine Angaben zum Trennungszeitpunkt gab.

5 Für 16 Fälle wurde eine Prozesszeit von einem Monat imputiert. 
hörigkeit, wodurch die Intensität der religiösen Bindung realistischer erfasst wird. Es ist davon auszugehen, dass streng gläubige Personen im Vergleich zu nicht religiösen Menschen nach einer gescheiterten Ehe die Aufnahme einer nachehelichen Partnerschaft ablehnen bzw. viel weiter zeitlich aufschieben. Da für religiöse Menschen eine Scheidung mit negativen sozialen Sanktionen verbunden sein kann, so würden sich diese durch das verfrühte Zusammenziehen mit dem neuen Partner eher noch verstärken (Wu/Balakrishan 1994). Die Berücksichtigung der Kontrollvariablen Wohnregion dient dazu, auf den Einfluss der Größe des Wohnortes zu schließen und wird anhand der Ausprägungen ,ländlich“ (unter 20.000 Einwohner), „städtisch“ (unter 100.000 Einwohner) und „Großstadt“ (mehr als 100.000 Einwohner) gemessen. Dabei ist zu beachten, dass die Angabe des Wohnsitzes zum Zeitpunkt des Interviews erfolgte. Somit können keine Aussagen darüber getroffen werden, ob sich die Person beispielsweise erst nach der Scheidung vom ehemaligen Partner für den Umzug in eine andere Wohnregion entschieden hat. Es ist vorstellbar, dass mit steigendem Urbanitätsgrad auch eine Erhöhung der Realisierung nachehelicher Partnerschaften einhergeht, da ein städtisches Umfeld vielfältigere Möglichkeiten für das Zusammentreffen von potenziellen Partnern bietet.

\section{Empirische Analysen}

\subsection{Deskriptive Ergebnisse}

Hinsichtlich des zeitlichen Verlaufs zum Übergang in eine nacheheliche Partnerschaft werden anhand deskriptiver Schätzungen nur geringfügige geschlechtsspezifische Unterschiede $^{6}$ sichtbar (Abbildung 1). In den ersten drei Jahren überlagern sich die Kurven nahezu, und der steile Abfall bestätigt, dass eine „Wiederverpartnerung“ bei geschiedenen Männern und Frauen unmittelbar nach der Auflösung der Ehe besonders hoch ist: immerhin erleben 25 Prozent dieses Ereignis innerhalb der ersten zwei Jahre. Eine Erklärung dafür könnte sein, dass der Auslöser für die Trennung oft ein neuer Partner ist und das $\mathrm{Zu}$ sammenziehen mit diesem Partner schon vor der rechtsgültigen Scheidung erfolgt. Betrachtet man den Verlauf der Kurven insgesamt, so zeigen sich die stärksten Unterschiede zwischen Männer und Frauen im Zeitraum von 36 bis 48 Monaten. Dementsprechend gehen im dritten Jahr nach der Scheidung Männer etwas häufiger als Frauen eine nacheheliche Partnerschaft ein. Zum Ende des Beobachtungszeitraums (nach 15 Jahren) haben 38 Prozent der Männer und 41 Prozent der Frauen das Ereignis noch immer nicht erfahren.

6 Teststatistik: $\log -\mathrm{Rank}=0.347$; Wilcoxon $=0.350$ 
Abbildung 1: Kaplan-Meier-Survivalkurve zum Übergang in eine nacheheliche Partnerschaft für geschiedene Männer und Frauen

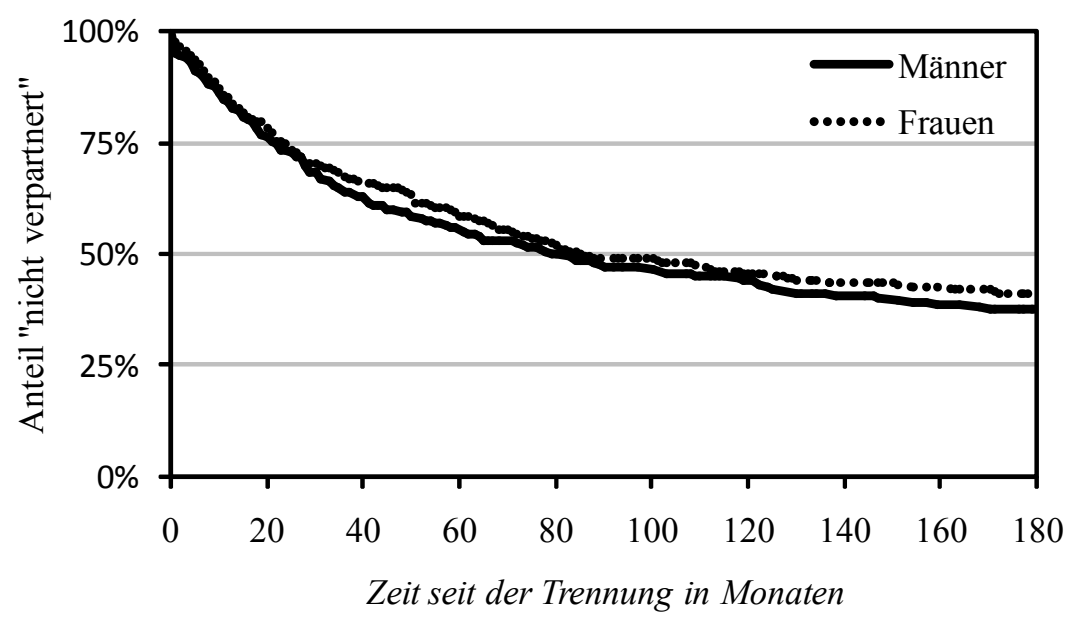

Quelle: Generations and Gender Survey 2005, Deutschland (eigene Berechnungen)

\subsection{Ergebnisse der multivariaten Schätzung}

In Tabelle 1 sind die Ergebnisse der ereignisanalytischen Modelle dargestellt. Die zuvor sichtbare Zeitabhängigkeit des „Wiederverpartnerungsprozesses“ wird in der multivariaten Schätzung durch eine angemessene Spezifikation der Baseline in sieben relativ kurze Zeitintervalle modelliert. Der Einfluss der Dauer seit der Trennung auf den Übergang zur nachehelichen Partnerschaft ist signifikant und erweist sich über alle Modelle hinweg konstant. Das Risiko für die Aufnahme einer nachehelichen Partnerschaft ist unmittelbar nach der Trennung innerhalb des ersten Jahres am höchsten. Im Zeitraum von ein bis zwei Jahren verringert sich das Risiko nur geringfügig um ungefähr 16 Prozent. Nach dem dritten Jahr ist das Muster der relativen Risiken zum Verlauf der Verweildauer von einem starken Abfall gekennzeichnet. Im nächsten Zeitintervall (5 bis 7 Jahre) wird dieser Trend durch eine Erholungsphase unterbrochen, jedoch fällt im darauffolgenden Zeitintervall das Risiko, das Ereignis zu erfahren, sehr stark ab. Bezugnehmend auf die Referenzkategorie ist nach mehr als zehn Jahren das Risiko um 85 Prozent geringer. Zusammenfassend lässt sich sagen, dass mit zunehmender Prozesszeit das Risiko für eine nacheheliche Partnerschaft immer weiter abnimmt.

Der Einfluss des Bildungsniveaus folgt dem prognostizierten Zusammenhang. Dementsprechend zeigen Personen mit hohem Bildungsniveau auch die höchste Neigung in Bezug auf das nacheheliche Paarbildungsverhalten. Zieht man die Werte aus dem dritten Modell ${ }^{7}$ heran, so ist für Hochgebildete ein fast 35 Prozent höheres Risiko im Vergleich zu niedrig Gebildeten ausgewiesen. Auch Personen mit mittlerer Bildung haben gegen-

7 Anhand des Log-Likelihood-Wertes kann für dieses Modell die höchste Güte abgelesen werden 
über der Referenzgruppe bessere Chancen zur Gründung einer Folgepartnerschaft, selbst wenn diese Unterschiede etwas geringer ausfallen und statistisch nicht signifikant sind.

Die ermittelten Koeffizienten für den Einfluss der Religiosität zeigen, dass eine starke Bindung zur Religion sich negativ auf den Übergangsprozess auswirkt. Zwischen „etwas religiösen“ und „nicht religiösen“ Menschen sind keine wesentlichen Unterschiede zu erkennen. Jedoch muss bei der Interpretation dieses Ergebnisses beachtet werden, dass bei gläubigen Menschen im Allgemeinen eine geringere Scheidungsneigung vorhanden ist (Böttcher 2006). Dadurch ist die Gruppe der sehr gläubigen Befragten stark selektiv, weil sich nur ein geringer Anteil überhaupt scheiden lässt und somit erst in die Risikopopulation gelangt.

Führt man sich den Einfluss der Wohnortgröße vor Augen, so wirkt sich das Leben in ländlichen Gebieten erstaunlicherweise positiv aus, die Übergangsrate ist im Vergleich zu Großstädtern etwa um 40 Prozent höher. In Bezug auf diesen Zusammenhang ist ein Selektionseffekt denkbar. In diesem Sinne könnte unterstellt werden, dass sich Menschen, die auf dem Land leben, vielleicht nur dann trennen bzw. scheiden lassen, wenn sie bereits wieder einen neuen Partner in Aussicht haben. Dieser Befund könnte auch durch einen in Großstädten höheren Anteil an Partnerschaften ohne gemeinsamen Haushalt zustande kommen. Da die Information zum Wohnort nur zum Zeitpunkt des Interviews vorliegt, könnte das Ergebnis auch durch den Wegzug von Geschiedenen aus ländlichen Gebieten beeinflusst sein.

Betrachtet man den Einfluss, den das Alter des jüngsten Kindes ausübt, so geht mit höherem Alter der Kinder eine geringe Neigung zur nachehelichen Paarbildung einher, entgegen der angestellten Vermutung, dass kleinere Kinder eine erneute Haushaltsgründung behindern (Modell 1). Für Personen mit Kinder über 18 Jahre ist das Risiko um mehr als die Hälfte vermindert, verglichen mit Personen mit Kindern bis zu neun Jahren. Da aber dieser Effekt im zweiten Modell durch die Kontrolle der Variablen Alter bei Trennung überlagert wird, kann hier folgende Abhängigkeit zwischen den beiden Einflussgrößen vermutet werden: Im Falle eines fortgeschrittenen Alters bei Trennung sind die Kinder zum Zeitpunkt der Auflösung der Ehe höchstwahrscheinlich auch älter. Im dritten Modell zeigt sich der Einfluss der Anzahl der Kinder im Haushalt wie in der dazu formulierten Hypothese. Für Personen, die im Haushalt zwei und mehr Kinder zum Zeitpunkt der Trennung haben, ist das Risiko für das Zusammenziehen mit einem neuen Partner um 20 Prozent geringer als für Befragte mit nur einem Kind im Haushalt. Eine Einschränkung ergibt sich daraus, dass dieser Befund nicht gleichermaßen für Frauen und Männer gedeutet werden kann, und zwar wegen der ungleichen Aufteilung der Kinder auf die getrennten Haushalte, in den meisten Fällen zugunsten des mütterlichen Haushaltes (BMFSFJ 2003: 8).

Die geschätzten Koeffizienten hinsichtlich der Variablen Alter bei Trennung folgen der abgeleiteten Hypothese, dass sich im höheren Alter die Chancen zur Gründung einer nachehelichen Partnerschaft signifikant verschlechtern. Bezugnehmend auf das dritte Modell sinkt das Risiko um 39 Prozent, wenn das Trennungsalter zwischen 35 und 49 Jahren liegt, bei über 50 Jahren sogar um 62 Prozent im Vergleich zur Referenzaltersgruppe 25 bis 34 Jahre. Durch die enorme Verbesserung der Modellgüte kann dem Alter bei Trennung eine hohe statistische Relevanz beigemessen werden. 
Tabelle 1: Relative Risiken des Übergangs in eine nacheheliche Partnerschaft, Ergebnisse des Piecewise-Constant-Exponentialmodell

\begin{tabular}{|c|c|c|c|}
\hline & Modell 1 & Modell 2 & Modell 3 \\
\hline \multicolumn{4}{|l|}{ Dauer in Monaten } \\
\hline$<12$ & 1 & 1 & 1 \\
\hline $12-24$ & 0,83 & 0,84 & 0,84 \\
\hline $24-36$ & $0,73 * *$ & $0,74 *$ & $0,74 * *$ \\
\hline $36-60$ & $0,41 * * *$ & $0,42 * * *$ & $0,42 * * *$ \\
\hline $60-84$ & $0,45 * * *$ & $0,46 * * *$ & $0,46 * * *$ \\
\hline $84-120$ & $0,21 * * *$ & $0,22 * * *$ & $0,22 * * *$ \\
\hline$>120$ & $0,15 * * *$ & $0,15 * * *$ & $0,15 * * *$ \\
\hline \multicolumn{4}{|l|}{ Geschlecht } \\
\hline Männer & 1 & 1 & 1 \\
\hline Frauen & 0,97 & 0,89 & 0,97 \\
\hline \multicolumn{4}{|l|}{ Bildung } \\
\hline niedrig & 1 & 1 & 1 \\
\hline mittel & 1,18 & 1,16 & 1,16 \\
\hline hoch & $1,30 *$ & $1,38 *$ & $1,35 *$ \\
\hline \multicolumn{4}{|l|}{ Religiosität } \\
\hline sehr religiös & 1 & 1 & 1 \\
\hline etwas religiös & $1,34 *$ & $1,29 *$ & $1,30 *$ \\
\hline nicht religiös & $1,31 *$ & 1,21 & 1,22 \\
\hline \multicolumn{4}{|l|}{ Wohnregion } \\
\hline Großstadt & 1 & 1 & 1 \\
\hline städtisch & 1,00 & 1,01 & 1,01 \\
\hline ländlich & $1,42 * *$ & $1,39 *$ & $1,42 *$ \\
\hline \multicolumn{4}{|l|}{ Alter des jüngsten Kindes } \\
\hline kein Kind & 1,04 & 1,03 & - \\
\hline 0-9 Jahre & 1 & 1 & - \\
\hline 10-17 Jahre & 0,80 & 1,30 & - \\
\hline 18 und älter & $0,47 * * *$ & 1,07 & - \\
\hline \multicolumn{4}{|l|}{ Anz. der Kinder im Haushalt } \\
\hline kein Kind & - & - & 0,95 \\
\hline 0 Kinder im $\mathrm{HH}$ & - & - & 1,12 \\
\hline $1 \mathrm{Kind}$ im $\mathrm{HH}$ & - & - & 1 \\
\hline $2+$ Kinder im HH & - & - & $0,79 *$ \\
\hline \multicolumn{4}{|l|}{ Alter bei Trennung } \\
\hline $18-24$ & - & $1,24 *$ & $1,23 *$ \\
\hline $25-34$ & - & 1 & 1 \\
\hline $35-49$ & - & $0,55 * * *$ & $0,61 * * *$ \\
\hline $50+$ & - & $0,36 * * *$ & $0,38 * * *$ \\
\hline Log Likelihood & $-1274,35 \quad \mathrm{~N}=847$ & $-1253,308 \mathrm{~N}=847$ & $-1251,658 \quad \mathrm{~N}=84$ \\
\hline
\end{tabular}

$* * * \mathrm{p} \leq 0.01 * * 0.01<\mathrm{p} \leq 0.05 * 0.05<\mathrm{p} \leq 0.1$

Anmerkung: bei Fällen ohne oder fehlerhafte Angabe ist eine zusätzliche Kategorie "missing" eingegangen

Quelle: Generations and Gender Survey 2005, Deutschland (eigene Berechnungen). 


\subsection{Interaktionseffekte}

Da in der multiplikativen Modellschätzung kein signifikanter ${ }^{8}$ Einfluss der Variablen Geschlecht sichtbar ist, wird im Folgenden mit der Berechnung von Interaktionseffekten die Geschlechterabhängigkeit in Bezug auf die Haupteinflussfaktoren näher spezifiziert.

Abbildung 2: Interaktion von Geschlecht und Alter bei Scheidung (a) und von Geschlecht und Bildungsniveau (b), relative Risiken (standardisierte Darstellung)

a

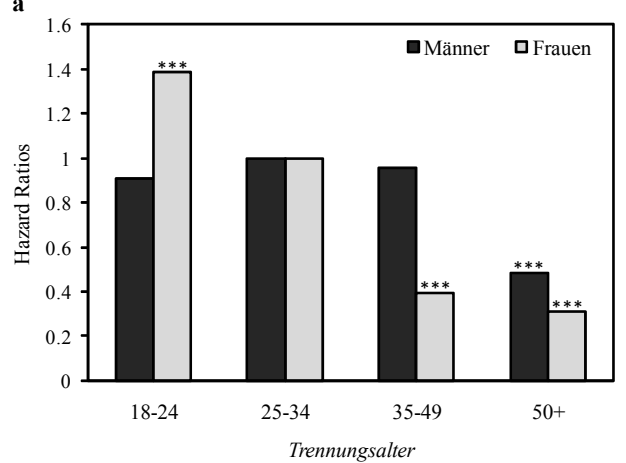

b

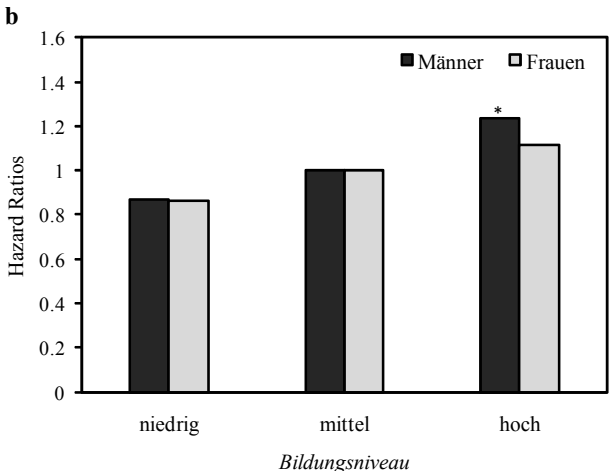

Anmerkung: Weitere Kontrollvariablen im Modell a sind Dauer nach Trennung, Bildung, Religiosität, Wohnregion und Anzahl der Kinder im Haushalt; im Modell b sind alle genannten Kontrollvariablen einbezogen - nur ist anstelle von Bildung das Trennungsalter enthalten.

$* * * \mathrm{p} \leq 0.01 * * 0.01<\mathrm{p} \leq 0.05 * 0.05<\mathrm{p} \leq 0.1$

Quelle: Generations and Gender Survey 2005, Deutschland (eigene Berechnungen)

Wie in Abbildung 2a deutlich erkennbar ist, kommt der negative Effekt des Alters bei Frauen viel stärker als bei Männern zum Tragen. Bei Frauen ist im Vergleich zu der jüngeren Referenzaltersgruppe 25-34 ein sehr starkes Absinken des Risikos einer nachehelichen Paarbildung bereits in der Alterskategorie 35-49 erkennbar. Dagegen wirkt sich der Alterseffekt bei Männern erst in der Altersgruppe 50+ hemmend aus und bleibt über die drei jüngeren Altersgruppen relativ gleich. Auffallend ist, dass bei den jungen Frauen (1824) die Neigung zur „Wiederverpartnerung“ am höchsten ist. Dieser signifikante Befund dürfte zum einen daran liegen, dass der Beginn der relevanten Partnerschaftskarriere bei Frauen allgemein in etwas jüngerem Alter als bei den männlichen Altersgenossen stattfindet. Wenn Männer schon zum Zeitpunkt der Heirat und somit auch bei der Scheidung älter als ihre Partnerinnen sind, verschiebt sich bei ihnen dementsprechend auch die Gründung eines nachehelichen Haushalts im Lebenslauf weiter nach hinten. Vergleicht man die Übergangsraten der ältesten Personen, so ist für Männer im fortgeschrittenen Alter (50+) ein um 52 Prozent, für Frauen ein um 69 Prozent verringertes Risiko zu ver-

8 Es kann trotzdem ein tatsächlicher Effekt vorliegen, auch wenn die Ergebnisse des statistischen Tests nicht signifikant sind. Außerdem können anhand der statistischen Signifikanz keine Aussagen über die Stärke eines Effekts getroffen werden (Schnell et al. 2005: 452f.). 
zeichnen. Ob diese hochsignifikanten Ergebnisse vorrangig durch partnermarktspezifische Ungleichgewichte zustande kommen oder Frauen in höherem Alter tatsächlich ein bewusst vermindertes Paarbildungsverhalten aufweisen, lässt sich anhand dieser Untersuchung leider nicht aufklären.

In Bezug auf geschlechtsspezifische Bildungseffekte konnte mit der Berechnung des in Abbildung $2 \mathrm{~b}$ dargestellten Interaktionseffekts festgestellt werden, dass sich mit steigendem Bildungsabschluss die Neigung zur nachehelichen Paargründung sowohl für Männer als auch für Frauen erhöht. Im Vergleich zu Personen mit mittlerer Bildung haben sowohl Männer und Frauen mit niedriger Bildung ein gleichermaßen geringeres Risiko der Paarbildung. Nur innerhalb der Kategorie hohes Bildungsniveau ist eine geringfügig höhere Paarbildungsneigung bei den Männern sichtbar. Aufgrund der mangelhaften statistischen Signifikanz dieser Befunde, können jedoch nur Vermutungen angestellt werden. Einerseits könnte sich für gut ausgebildete Frauen eine stärkere Motivation ergeben, die Entscheidung für eine neue Partnerschaft weiter aufzuschieben, da sie weniger von einem männlichen Verdiener abhängig sind. Anderseits könnte sich für sehr hochgebildete Frauen ein hinderlicher Aspekt daraus ergeben, dass mitunter nicht genügend geeignete männliche Partner mit vergleichbarem Bildungsniveau zur Verfügung stehen, weil sie entweder altersbedingt nicht mehr vorhanden oder partnerschaftlich bereits gebunden sind (Lankuttis/Blossfeld 2003: 17).

Aufgrund der Problematik, dass der Einfluss des Alters des jüngsten Kindes durch den stark signifikanten Alterseffekt überlagert wird, ist es sinnvoll, diese Einflussgröße, im Verlauf der Prozesszeit, separat in einem Interaktionsmodell zu überprüfen. Dazu werden getrennte Modellrechnungen für Männer und Frauen vorgenommen. Ein weiterer Grund für die Betrachtung des Alters des jüngsten Kindes besteht darin, so eine generelle Wirkung zum Einfluss von Kindern bei Männern nachzuweisen, da die Variable Anzahl der Kinder im Haushalt aufgrund der mangelnden Besetzungszahlen für Männer nicht herangezogen werden kann.

Abbildung 3: Interaktion von Dauer nach der Trennung und Alter des jüngsten Kindes, relative Risiken (getrennte Darstellung für Männer und Frauen)
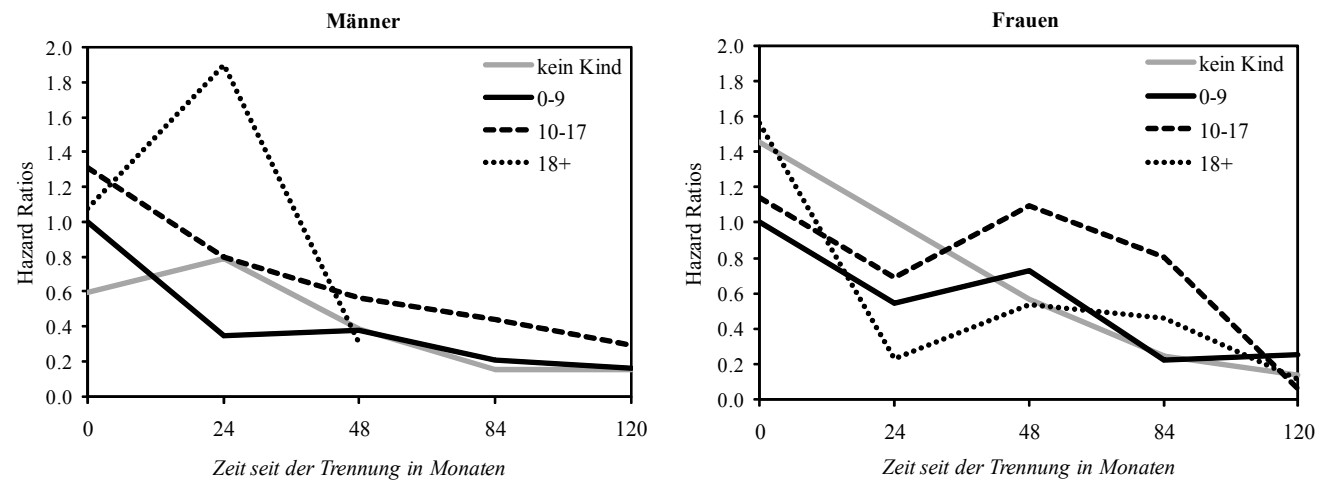

Anmerkung: Weitere Kontrollvariablen in beiden Modellen sind Bildung, Religiosität, Wohnregion und Trennungsalter.

Quelle: Generations and Gender Survey 2005, Deutschland (eigene Berechnungen) 
Wie Abbildung 3 verdeutlicht, haben sowohl Männer als auch Frauen mit Kindern im Alter von 10 bis 17 zum Zeitpunkt der Trennung eine höhere Übergangsrate für das $\mathrm{Zu}$ sammenziehen mit einem neuen Partner als geschiedene Männer und Frauen mit kleineren Kindern unter zehn Jahren. Dieser Risikoverlauf ist ein Indiz dafür, dass jüngere Kinder, die relativ hohe Betreuungsansprüche stellen, eine Barriere für das nacheheliche Paarbildungsverhalten darstellen können. Demnach könnte auch für geschiedene Väter, die durch kleinere Kinder eine stärkere Bindung an die ehemalige Familie haben, eine nacheheliche Haushaltsgründung erschwert sein. Die Bestätigung dieses vermuteten $\mathrm{Zu}$ sammenhangs zum negativen Einfluss von kleinen Kindern - unabhängig davon, ob sie im mütterlichen oder väterlichen Haushalt leben - ist aufgrund der geringen Fallzahlen jedoch mit Einschränkungen verbunden. Hinsichtlich der Signifikanz der Ergebnisse sollten nur die Werte für das erste Zeitintervall, also innerhalb der ersten 24 Monate herangezogen werden.

Eine nennenswerte Auffälligkeit in dieser Darstellung ist jedoch das starke Absinken des Risikos für Geschiedene ohne Kinder im Zeitverlauf. Gerade für diese Personengruppe wäre zu jedem Zeitpunkt eine höhere Neigung zur nachehelichen Paarbildung zu erwarten, weil diese beispielsweise die Realisierung eines eventuellen Kinderwunsches ermöglichen würde. Scheinbar ist die Dynamik zur nachehelichen Paarbildung für Geschiedene ohne Kinder jedoch primär auf den Beginn der Nachscheidungsdauer gelegt. Somit könnte man vermuten, dass bei Geschiedenen ohne Kinder eine Art Selektionseffekt die immer weiter absinkende Neigung im Zeitverlauf erklärt. Einige Geschiedene vermeiden möglicherweise das Zusammenziehen mit einem Partner, weil sie sich bewusst für das Alleinleben und gegen eine Familie entscheiden. Dieser Befund zeigt sich bei Männern und Frauen nicht gleichermaßen. Kinderlose Frauen weisen unmittelbar nach der Scheidung noch eine sehr hohe Übergangsrate auf und erst im Zeitverlauf sinkt diese unter das Niveau geschiedener Mütter. Daher scheint die geringere Familienorientierung bei kinderlosen Männern stärker ausgeprägt, da die Übergangsrate niedriger im Vergleich zu geschiedenen Männern mit Kindern ist.

\section{Zusammenfassung und Ausblick}

Die Zielsetzung dieser Studie bestand darin, den Einfluss demografischer und sozioökonomischer Determinanten des nachehelichen Paarbildungsprozesses zu analysieren, und zwar mit Schwerpunkt auf geschlechtsspezifische Abhängigkeiten. Bei der Gründung einer Nachfolgebeziehung fällt die Intensität besonders am Beginn des Prozesses (in den ersten 24 Monaten) bei Männern und Frauen gleichermaßen sehr hoch aus. Dieses Ergebnis passt zu den Befunden in Bezug auf den Wiederheiratsprozess. Lankuttis und Blossfeld (2003) stellen fest, dass die Wiederheiratsneigung zwei bis fünf Jahre nach der Trennung am höchsten ist. Damit ist eine logische Reihenfolge in der zeitlichen Intensität der Ereignisse gegeben. Eine hohe Neigung zur nachehelichen Paarbildung ist der Wiederheiratsneigung vorangestellt. Die Erklärung, warum nacheheliche Partnerschaften oft unmittelbar nach der Trennung erfolgen, kann letztlich darauf zurückgeführt werden, dass einige Scheidungen erst durch das Kennenlernen eines neuen Partners verursacht werden. Dieser Aspekt scheint für die nacheheliche Paarbildung von enormer Bedeutung zu sein, leider 
ist die Prüfung des vermuteten Zusammenhangs hinsichtlich der Kausalität mit den vorliegenden Daten nicht möglich.

Mit zunehmender Verweildauer verringerte sich die Übergangsrate, wobei an dieser Stelle das Zusammenspiel interagierender Faktoren angeführt werden kann. Je länger eine Person im Ausgangszustand „geschieden“ verweilt, desto älter wird sie und desto mehr sinken die Chancen für die Aufnahme einer nachehelichen Partnerschaft. Dieser festgestellte negative Alterseffekt ist konsistent mit der eingangs formulierten Hypothese und auch ein replizierter Befund bei der Untersuchung von Folgebeziehungen (Klein 1990; Bumpass/Sweet/Martin 1990; Wu/Balakrishan 1994; Wu/Schimmele 2005).

Betrachtet man die festgestellten Unterschiede zwischen den einzelnen Altersgruppen im Kontext strukturbedingter Mechanismen der Partnerwahl, so scheinen Geschiedene in höherem Alter schlechtere Aussichten zu haben, mit einem neuen Partner zusammenzuziehen. Für Frauen ist dieser Effekt deutlich stärker ausgeprägt, was auf die Verengung des Partnermarkts zuungunsten älterer Frauen hindeutet. Hinsichtlich der untersuchten Bildungseinflüsse bestätigt sich die Vermutung, dass mit der Höhe des Bildungsniveaus tendenziell eine steigende Neigung zur nachehelichen Paarbildung einhergeht. Da sich dieser positive Effekt sowohl für Männer als auch für Frauen zeigte, sind gemäß den Annahmen der Familienökonomie traditionell orientierte Familienmuster im Sinne einer geschlechtsspezifischen Arbeitsteilung beim nachehelichen Paarbildungsprozess offenbar weniger von Bedeutung. Vielmehr können die Bedingungen für das Zustandekommen einer nachehelichen Partnerschaft von höher ausgebildeten Personen verbessert werden. Verallgemeinernd lässt sich daraus schlussfolgern, dass die eigene ökonomische Unabhängigkeit ein ausschlaggebendes Kriterium in Bezug auf die Attraktivität eines potenziellen Partners ist.

In vielen Studien wurde die Existenz von gemeinsamen Kindern aus der vorhergehenden Ehe als entscheidender Einflussfaktor für den „Wiederverpartnerungsprozess“ von Geschiedenen beschrieben (Ermisch 2002; Lampard/Peggs 1999; Bernhardt 2000; Hughes 2000). Inwieweit das Vorhandensein von Kindern aus einer vorhergehenden Ehe den Übergang in eine nacheheliche Partnerschaft behindert, konnte auf Basis der hier vorgelegten Analysen nur anhand der Kinder im Haushalt zum Zeitpunkt der Trennung eindeutig festgestellt werden. Leben zwei und mehr Kinder im Haushalt, so weist diese Person eine geringere Übergangsrate auf. Der weiterhin vermutete Zusammenhang, dass kleinere Kinder einen hemmenden Einfluss auf die Höhe der Übergangsrate haben, kristallisierte sich durch die Berechnung des Interaktionseffektes zum Alter des jüngsten Kindes in Verbindung mit der Dauer seit der Trennung heraus. Geschiedene Männer und Frauen mit Kindern unter 10 Jahren zeigen eine geringere Übergangsrate als geschiedene Elternteile mit Kindern im jugendlichen Alter.

Zur Vertiefung des nachehelichen Paarbildungsverhaltens sind weitere Studien im Bereich der Familienforschung angebracht, da diese Phase der Familienbiografie aufgrund kontinuierlich hoher Scheidungszahlen immer mehr an Bedeutung gewinnen wird. Künftige Untersuchungen sollten unter anderem die Unterhaltsproblematik näher beleuchten, da bisherige Studien hier ambivalente Ergebnisse hervorbrachten (Engstler 2003; De Graaf/Kalmijn 2003). Ein weiterer relevanter Aspekt ist die Erweiterung bildungsabhängiger Faktoren um den Einfluss der Erwerbstätigkeit, um so die finanzielle Unabhängigkeit beurteilen zu können. Dieser Umstand sollte in weiterführenden Analysen unbedingt 
als zeitabhängiger Faktor betrachtet werden. Schließlich stellt sich besonders im Hinblick auf geschlechtsspezifische Unterschiede die Frage, ob eine Frau erst nach der Scheidung wieder eine Erwerbsarbeit zur Überwindung der negativen Scheidungsfolgen aufnimmt und in diesem Fall weniger auf eine nacheheliche Haushaltsgründung angewiesen ist. Insgesamt lässt sich anhand der vorausgegangenen Analysen schlussfolgern, dass sich das Paarbildungsverhalten von Männern und Frauen weniger stark als zunächst angenommen unterscheidet. Nur im Hinblick auf den Einfluss des Trennungsalters haben die Ergebnisse gravierende Unterschiede zwischen Männer und Frauen aufgedeckt.

\section{Danksagung}

An erster Stelle möchte ich mich bei Michaela Kreyenfeld für die tatkräftige Unterstützung bei der Erstellung dieses Aufsatzes bedanken. Ich bedanke mich außerdem bei Esther Geisler, Anne Hornung und Karin Tesching für wertvolle Hinweise und Anregungen sowie bei Werner Richter für die sorgfältige Durchsicht dieses Manuskriptes. Ausdrücklicher Dank gilt den beiden anonymen Gutachtern für die vielen Verbesserungsvorschläge und alternativen Sichtweisen.

\section{Literatur}

Akers, D. S. (1967). On measuring the marriage squeeze. Demography, 4, S. 907-925.

Blau, P. M. (1967). Exchange and power in social life. New York: Wiley ( $2^{\text {nd }}$ printing).

Blau, P. M. (1977). Macrosociological theory of social structure. American Journal of Sociology, 83 (1), S. $26-54$

Blau, P. M. (1994). Structural contexts of opportunities. Chicago: University of Chicago Press.

Blossfeld, H.-P. \& Rohwer, G. (2002). Techniques of event history modeling: New approaches to causal analysis. Mahwah, N.J., London: Lawrence Erlbaum Associates

Blossfeld, H.-P., Rohwer, G. \& Golsch, K. (2007). Event history analysis with Stata. Mahwah, N.J., London: Lawrence Erlbaum Associates.

Beck-Gernsheim, E. (1994). Auf dem Weg in die postfamiliale Familie - Von der Notgemeinschaft zur Wahlverwandtschaft. In: Beck, U. \& Beck-Gernsheim, E. (Hrsg.), Riskante Freiheiten. Individualisierung in modernen Gesellschaften. Frankfurt am Main: Suhrkamp, S. 115-138.

Becker, G. S. (1973). A theory of marriage: Part I. The Journal of Political Economy, 81 (4), S. 813-846.

Becker, G. S. (1993). A treatise on the family. Harvard University Press (Enlarged edition).

Bernhardt, E. M. (2000). Repartnering among Swedish men and women: A case study emerging patterns in the second demographic transition. (Paper contributed to the FFS Flagship conference, Brussels, 29-31 May 2000).

Booth, A. \& Edwards, J. N. (1992). Starting over: Why remarriages are more unstable. Journal of Family Issues, 13 (2), S. 179-194.

Böttcher, K. (2006). Scheidung in Ost- und Westdeutschland. Der Einfluss der Frauenerwerbstätigkeit auf die Ehestabilität. Kölner Zeitschrift für Soziologie und Sozialpsychologie, 58 (4), S. 592-616.

Bumpass, L., Sweet, J. \& Martin, T. C. (1990). Changing patterns of remarriage. Journal of Marriage and the Family, 52 (3), S. 747-756.

Bundesministerium für Familie, Senioren, Frauen und Jugend (BMFSFJ) (2003). Wenn aus Liebe rote Zahlen werden - über die wirtschaftlichen Folgen von Trennung und Scheidung. Bonn: BMFSFJ.

Bundeszentrale für politische Bildung (Hrsg.) (2008). Datenreport 2008. Ein Sozialbericht für die Bundesrepublik Deutschland. Bonn: Bundeszentrale für politische Bildung 
Daly, M. (2005). Changing family life in Europe: Significance for state and society. European Societies, 7 (3), S. 379-398.

De Graaf, P. M. \& Kalmijn, M. (2003). Alternative routes in the remarriage market: Competing-risk analysis of union formation after divorce. Social Forces, 81 (4), S.159-1498.

Diekmann, A. (2004). Empirische Sozialforschung: Grundlagen, Methoden, Anwendungen. 11. Reinbek bei Hamburg: Rowohlt Taschenbuch Verlag (11. Auflage).

Engstler, H. (2003). Wiederheirat nach Scheidung. Trends und Faktoren der Wiederheiratshäufigkeit Geschiedener in der Schweiz. In: Dorbitz, J. \& Otto, J. (Hrsg.), Familienpolitik und Familienstrukturen. Materialien zur Bevölkerungswissenschaft, (Heft 108), S. 201-214.

Ermisch, J. (2002). Trying again: Repartnering after dissolution of a union. Colchester: University of Essex (Working papers of the Institute for Social and Economic Research, paper 2002-19).

Hochschild, A. R. (1997). The time bind: When work becomes home and home becomes work. New York: Metropolitan Books.

Heekerens, H.-P. (1988). Die zweite Ehe: Wiederheirat nach Scheidung und Verwitwung. Weinheim: Deutscher Studienverlag.

Hughes, J. (2000). Repartnering after divorce. Marginal mates and unwedded women. Australian Institute of Family Studies, Family Matters, 55, S. 16-21.

Klein, T. (1990). Wiederheirat nach Scheidung in der Bundesrepublik. Eine empirische Überprüfung bislang vorliegender Theorieansätze aus der Perspektive des Lebensverlaufs. Kölner Zeitschrift für Soziologie und Sozialpsychologie, 42 (1), S. 60-80.

Klein, T. (1999). Partnerwahl in Ehen und Nichtehelichen Lebensgemeinschaften. In: Klein, T. \& Lauterbach, W. (Hrsg.), Nichteheliche Lebensgemeinschaften. Analysen zum Wandel partnerschaftlicher Lebensformen. Opladen: Leske + Budrich, S. 207-234.

Klein, T. (2000). Partnerwahl zwischen sozialstrukturellen Vorgaben und individueller Entscheidungsautonomie. Zeitschrift für Soziologie der Erziehung und Sozialisation, 40, S. 229-243.

Lampard, R. \& Peggs, K. (1999). Repartnering: The relevance of parenthood and gender to cohabitation and remarriage among formerly married. British Journal of Sociology, 50 (3), S. 443-465.

Lankuttis, T. \& Blossfeld, H.-P. (2003). Determinanten der Wiederheirat nach der ersten Scheidung in der Bundesrepublik Deutschland. Zeitschrift für Familienforschung, 15 (1), S. 5-24.

Lengerer, A. (2001). Wo die Liebe hinfällt - ein Beitrag zur „Geographie“ der Partnerwahl. In: Klein, T. (Hrsg.), Partnerwahl und Heiratsmuster. Sozialstrukturelle Voraussetzungen der Liebe. Opladen: Leske + Budrich, S. 133-162.

Lesthaeghe, R. (1992), Der zweite demographische Übergang in den westlichen Ländern: Eine Deutung. Zeitschrift für Bevölkerungswissenschaft, 18 (3), S. 313-354.

Nave-Herz, R. (2004). Ehe- und Familiensoziologie. Eine Einführung in Geschichte, theoretische Ansätze und empirische Befunde. Weinheim, München: Juventa.

Nye, F. I. (1979). Choice, exchange, and the family. In: Burr, W. R et al. (Hrsg.), Contemporary theories about the family. Vol. II. New York, London: Free Press, S. 1-41.

Oppenheimer, V. K. (1988). A theory of marriage timing. The American Journal of Sociology, 94 (3), S. 563-591.

Oppenheimer, V. K. (1994). Women's rising employment and the future of the family in industrial societies. Population and Development Review, 20 (2). S. 293-342.

Perelli-Harris, B., Kreyenfeld, M. \& Kubisch, K. (2009). Manual for the cleaning of the German GGS. Rostock: Max Planck Institute for Demographic Research (MPIDR-Working paper 2010-08).

Ruckdeschel, K., Ette, A., Hullen, G. \& Leven, I. (2006). Generations and Gender Survey. Dokumentation der ersten Hauptbefragung in Deutschland. Wiesbaden: Bundesinstitut für Bevölkerungsforschung (Materialien zur Bevölkerungswissenschaft, Heft 121a).

Schnell, R., Hill, P. B. \& Esser, E. (2005). Methoden der empirischen Sozialforschung. München, Wien: Oldenbourg Verlag (7., völlig überarbeitete und erweiterte Auflage).

Schoen, R. (1983). Measuring the tightness of a marriage squeeze. Demography, 20, S. 61-78.

South, S. J. (1991). Sociodemographic differentials in mate selection preferences. Journal of Marriage and the Family, 53 (4), S. 928-940. 
Stauder, J. (2006). Die Verfügbarkeit partnerschaftlich gebundener Akteure für den Partnermarkt. Kölner Zeitschrift für Soziologie und Sozialpsychologie, 58 (4), S. 617-637.

Tammen, B. (2007). Familienrecht. In: Ecarius, J. (Hrsg.), Handbuch der Familie. Wiesbaden: VS Verlag für Sozialwissenschaften.

Textor, M. R. (2010). Stieffamilien. In: Staatsinstitut für Frühpädagogik (IFP) (Hrsg.). Das OnlineFamilienhandbuch. https://www.familienhandbuch.de/teil-und-stieffamilien/stieffamilien/stieffamilien [Stand: 2011-05-27].

Thibaut, J. W. \& Kelley, H. H. (1959). The social psychology of groups. New York: Wiley.

Wu, Z. \& Balakrishnan, T. R. (1994). Cohabitation after marital dissolution in Canada. Journal of Marriage and the Family 56, S. 723-734.

Wu, Z. \& Schimmele, C. M. (2005). Repartnering after first union disruption. Journal of Marriage and the Family 67, S. 27- 36.

Eingereicht am/Submitted on: 09.11.2009

Angenommen am/Accepted on: 19.07.2010

Anschrift der Autorin/Address of the author:

Ina Jaschinski, Diplom-Demographin

Statistik Austria

Guglgasse 13

1110 Wien

Österreich/Austria

E-Mail: ina.jaschinski@statistik.gv.at

\section{Anhang}

Tabelle A1: Anzahl der in die Analyse ein- und ausgeschlossenen Fälle

\begin{tabular}{|c|c|c|c|}
\hline & Gesamt & Männer & Frauen \\
\hline Fallzahl im GGS & 10.017 & 4.610 & 5.407 \\
\hline Ausschluss weil: & & & \\
\hline keine Scheidung & 8.717 & & \\
\hline Partner gestorben & 368 & & \\
\hline inkonsistente Reihenfolge der Ereignisse & 37 & & \\
\hline außerhalb der Altersspanne (18-65) & 3 & & \\
\hline k. A. zum Trennungs- und Scheidungszeitpkt. & 28 & & \\
\hline k. A. zum Zusammenzug mit neuem Partner & 17 & & \\
\hline Personen in der Stichprobe & 847 & $338(39,91 \%)$ & $509(60,09 \%)$ \\
\hline nacheheliche Partnerschaft & 485 & $201(40,53 \%)$ & $284(55,80 \%)$ \\
\hline keine nacheheliche Partnerschaft & 362 & $137(59,47 \%)$ & $225(44,20 \%)$ \\
\hline
\end{tabular}

Quelle: Generations and Gender Survey 2005, Deutschland (eigene Berechnungen) 
Tabelle A2: Verteilung der Ereignisse und der Risikozeit auf die Ausprägung der unabhängigen Variablen

\begin{tabular}{|c|c|c|c|c|c|c|}
\hline & \multicolumn{2}{|c|}{ Gesamt } & \multicolumn{2}{|c|}{ Männer } & \multicolumn{2}{|c|}{ Frauen } \\
\hline & Ereignisse & $\begin{array}{l}\text { Risikozeit } \\
(\%)\end{array}$ & Ereignisse & $\begin{array}{l}\text { Risikozeit } \\
\text { (\%) }\end{array}$ & Ereignisse & $\begin{array}{l}\text { Risikozeit } \\
(\%)\end{array}$ \\
\hline \multicolumn{7}{|l|}{ Geschlecht } \\
\hline Männer & 201 & 39.47 & & & & \\
\hline Frauen & 284 & 60.53 & & & & \\
\hline \multicolumn{7}{|l|}{ Bildung } \\
\hline niedrig & 52 & 13.75 & 13 & 8.79 & 39 & 16.99 \\
\hline mittel & 293 & 60.16 & 116 & 58.89 & 177 & 61.00 \\
\hline hoch & 135 & 25.31 & 70 & 31.50 & 65 & 21.27 \\
\hline missing & 5 & 0.77 & 2 & 0.83 & 3 & 0.74 \\
\hline \multicolumn{7}{|l|}{ Religiosität } \\
\hline gläubig religös & 56 & 15.26 & 23 & 9.33 & 33 & 19.12 \\
\hline etwas religiös & 230 & 45.85 & 81 & 45.78 & 149 & 45.90 \\
\hline nicht religiös & 195 & 38.51 & 93 & 44.21 & 102 & 34.79 \\
\hline missing & 4 & 0.38 & 4 & 0.68 & 0 & 0.19 \\
\hline \multicolumn{7}{|l|}{ Wohnregion } \\
\hline Großstadt & 335 & 74.78 & 134 & 77.41 & 201 & 73.07 \\
\hline städtisch & 84 & 17.12 & 41 & 16.46 & 43 & 17.56 \\
\hline ländlich & 66 & 8.10 & 26 & 6.13 & 40 & 9.37 \\
\hline \multicolumn{7}{|l|}{ Trennungsalter } \\
\hline $18-24$ & 99 & 12.21 & 26 & 13.46 & 73 & 11.39 \\
\hline $25-34$ & 235 & 40.78 & 83 & 38.68 & 152 & 42.16 \\
\hline $35-44$ & 111 & 30.12 & 69 & 27.82 & 42 & 31.62 \\
\hline $45+$ & 40 & 16.89 & 23 & 20.04 & 17 & 14.84 \\
\hline \multicolumn{7}{|c|}{ Alter des jüngsten Kindes } \\
\hline 0-9 Jahre & 259 & 48.48 & 93 & 45.82 & 166 & 50.75 \\
\hline 10-17 Jahre & 64 & 14.51 & 36 & 12.90 & 28 & 15.55 \\
\hline 18 Jahre und älter & 28 & 11.43 & 13 & 8.12 & 15 & 13.59 \\
\hline kein Kind & 134 & 25.26 & 59 & 33.16 & 75 & 20.11 \\
\hline \multicolumn{7}{|c|}{$\begin{array}{l}\text { Anzahl der Kinder im Haushalt } \\
\text { bei Auflösung der Ehe }\end{array}$} \\
\hline 0 Kinder im $\mathrm{HH}$ & 87 & 16.80 & 73 & 31.97 & 14 & 6.90 \\
\hline $1 \mathrm{Kind}$ im $\mathrm{HH}$ & 152 & 27.97 & 45 & 21.45 & 107 & 32.22 \\
\hline 2+ Kinder im $\mathrm{HH}$ & 112 & 29.98 & 24 & 13.42 & 88 & 40.77 \\
\hline \multirow[t]{2}{*}{ kein Kind } & 134 & 25.26 & 59 & 33.16 & 75 & 20.11 \\
\hline & 485 & 100.00 & 201 & 100.00 & 284 & 100.00 \\
\hline
\end{tabular}

Quelle: Generations and Gender Survey 2005, Deutschland (eigene Berechnungen). 